\title{
Viva la vaccine
}

\author{
World Immunization Week 2016 gives us a chance to take stock of the current vaccine landscape, \\ celebrate some notable successes made in recent years and face up to the challenges remaining in \\ closing the gap to ensure that the full benefits of immunization are extended to all infants worldwide.
}

This month saw the launch of the world's first public dengue vaccination programme in the Philippines, where over 1 million school children are to be inoculated. Dengue virus is estimated to infect more than 100,000 million people worldwide each year, leading to 50,000 cases of dengue haemorrhagic fever and 22,000 deaths. Dengvaxia is a tetravalent vaccine effective against all four dengue virus serotypes and is the culmination of a 20-year, £1.2-billion development process by Sanofi Pasteur. With the RTS,S malaria vaccine currently being considered for approval and an effective vaccine against Ebola virus also on the horizon, there are certainly several reasons to be cheerful as we enter World Immunization Week 2016 (24-30 April).

We are now more than halfway through the Decade of Vaccines envisioned by the World Health Assembly, laid out in the ambitious Global Vaccine Action Plan ${ }^{1}$ (GVAP). It therefore seems like a good time to check the scorecard for progress towards the primary goal of "extending by 2020 and beyond the full benefits of immunization to all people, regardless of where they are born, who they are, or where they live." Vaccinations prevent an estimated 2-3 million deaths each year, yet another 18.7 million infants worldwide are still missing out on even the most basic package of vaccines. The overwhelming majority of these children live in low- and middle-income countries. Initial success of GVAP was to be measured by at least 90 low- and middle-income countries introducing one or more new or underutilized vaccines, and by the elimination and eradication of measles, rubella, neonatal tetanus and polio. Unfortunately, as noted in the 2015 assessment by the strategic advisory group of experts in immunization ${ }^{2}$, we are not on course to meet the majority of these early targets. The one notable success is that, so far, 86 countries have added at least one vaccine to their immunization programme. However, while vaccination decreased deaths from measles by $79 \%$ between 2000 and 2014, there are still more than 100,000 measles deaths globally each year. For rubella, reported cases have declined by $95 \%$ from more than 670,000 in 2000 , to approximately 33,000 in 2014 . Deaths from neonatal tetanus still occur in 24 countries, although 10 of these are close to achieving elimination. For polio, eradication is tantalizingly close but has remained that way for much of the past decade. Thanks to the Global Polio Eradication Initiative, the disease is now endemic in just two countries (Afghanistan and Pakistan) and just 359 cases were reported in 2014. What a waste it would be if we fail now to eradicate this debilitating disease having come so close.

Success for GVAP in the longer term is to be determined by the expansion of immunization programmes to achieve $90 \%$ coverage at the national level (and no less than $80 \%$ coverage at the district level), initially using the diphtheria-tetanuspertussis (DTP3) vaccine as a marker, before expecting these levels of coverage for all vaccines in national programmes. Again, these goals are unlikely to be met by the end of the decade. The DTP3 vaccine coverage in half of the WHO regions was still some way short of the $90 \%$ mark as recently as 2014 . On the whole, global vaccine coverage appears to be holding steady rather than increasing as hoped, owing to a range of challenges.

The resources available for healthcare systems of many low- and middle-income countries are often extremely limited. As new and under-utilized vaccines are introduced into national immunization programmes, already over-burdened healthcare systems come under further pressure. This can only be met with increased funding for purchasing, storage and delivery of vaccines, as well as ensuring that adequate numbers of healthcare workers have been recruited and trained with the necessary knowledge and skills. Compounding these problems are the increasingly complex $\mathrm{R} \& \mathrm{D}$ and regulatory processes. Combined with high manufacturing costs, this has led to a dramatic increase in the cost of many vaccines over the past 15 years. The Global Alliance for Vaccines and Immunization (GAVI; http://www.gavi.org/), a public-private partnership launched in 2000 , has worked to lower the prices of new and under-utilized vaccines for low-income countries. However, even at the low prices negotiated by GAVI, the introduction of the pneumococcal conjugate vaccine, rotavirus vaccine and human papillomavirus vaccine has led the cost of delivering the full basic vaccine package to increase 68 -fold between 2001 and $2016^{3}$. For middle-income countries, and those transitioning out of the GAVI-supported pool, cost rises can be far higher.

Clearly, pharmaceutical companies need to receive sufficient return on investment to incentivize developing new vaccine candidates and taking them through to regulatory approval. However, this needs to be balanced with the need to avoid profiteering and to ensure fair pricing commensurate with available resources in a given country. More attention could also be given to developing products that are better suited to delivery in resource-poor settings. For example, most products in current use are kept in strict refrigeration conditions from the point of their manufacture all the way through to administration to a patient. This can be challenging in remote areas where electricity supply is variable or absent. However, some of these vaccines have a heat stability profile that does not require constant refrigeration; re-assessing the storage conditions required for existing vaccines and taking heat stability into account when developing new products could help to substantially lower costs for low- and middle-income countries.

As healthcare interventions go, vaccines are one of the most successful and costeffective, and have saved countless millions of lives. If predictions of the dawn of a post-antibiotic era are accurate, vaccination will become increasingly important. We must redouble our efforts to develop and introduce new vaccines against microorganisms that are becoming resistant to antimicrobials. A decade alone may not be sufficient; we need this to be the century of the vaccine.

\footnotetext{
References

1. Global Vaccine Action Plan 2011-2020 (WHO, 2013); http://go.nature.com/jsoZII

2. SAGE 2015 Assessment Report Of The Global Vaccine Action Plan (WHO, 2015); http://go.nature.com/1T1 GmN

3. The Right Shot: Bringing Down Barriers to Affordable and Adapted Vaccines (Médecins Sans Frontières, 2015); http://go.nature.com/VwhbZN
}

Corrected: 29 April 2016 


\section{Correction: Viva la vaccine}

Nature Microbiology 1, 16066 (2016); published 26 April 2016; corrected 29 April 2016

The original version of this Editorial incorrectly suggested that the DTP3 vaccine is currently available in only half of the WHO regions. The intention was to note that in 2014 DTP3 vaccine coverage was less than $90 \%$ in half of the WHO regions. This has been corrected in all versions of the article. 